"Following is the Accepted Version of the article

Šeruga, D. \& Nagode, M. (2018)

Comparative analysis of optimisation methods for linking material parameters of exponential and power models: An application to cyclic stress-strain curves of ferritic stainless steel

published by

Proceedings of the Institution of Mechanical Engineers, Part L: Journal of Materials: Design and Applications

which can accessed at

https://doi.org/10.1177/1464420718790829.

This article may be used for non-commercial purposes in accordance with SAGE's Green Open Access policy." 


\title{
Comparative analysis of optimisation methods for linking material parameters of exponential and power models: an application to cyclic stress-strain curves of ferritic stainless steel
}

\author{
Domen Šeruga $^{1}$ and Marko Nagode ${ }^{1}$
}

\begin{abstract}
The four most commonly used optimisation methods for linking the material parameters of an exponential ArmstrongFrederick and a power Ramberg-Osgood model are compared for given cyclic stress-strain curves of a ferritic stainless steel EN 1.4512. These methods are the damped Gauss-Newton method, the Levenberg-Marquardt method, the Downhill Simplex method and a genetic algorithm. Globally optimal material parameters are obtained by parallel searches within the methods. The methods are tested for cyclic curves at temperatures of 20,300, 650 and 850 ${ }^{\circ} \mathrm{C}$. The optimal values of material parameters and $R^{2}$ values are comparable, whereas the search paths, the numbers of steps to reach optimal solutions and the processing time of the methods differ.
\end{abstract}

\section{Keywords}

ferritic stainless steel, material parameters, optimisation, stress-strain curve, cyclic curve, temperature, nonlinear kinematic hardening

\section{Introduction}

Metals operating in a high temperature environment must withstand deteriorative processes such as thermomechanical fatigue, creep, corrosion and oxidation. ${ }^{1-4}$ Titanium stabilised extra mild $12 \%$ chromium ferritic stainless steel EN 1.4512, as a typical representative of modern ferritic stainless steels, is now more commonly used for the manufacture of automotive exhaust systems and parts of gas turbines. It ensures high creep and corrosion resistance, oxidation stability and good mechanical properties for a competitive price as compared to austenitic stainless steels which have been traditionally used in high temperature environments. ${ }^{3,5-8}$ Titanium stabilisation additionally improves its weldability and decreases the recrystallisation rate at high temperatures. However such materials operating under variable mechanical and thermal loadings will still be subjected to thermomechanical fatigue, due to repeated phases of start-up, interchanges of full and partial load, and shutdown. In order to prevent failure due to thermomechanical fatigue during the design lifetime, damage prediction is becoming a necessary step in the early development stages of new components. ${ }^{3}$ The damage predictions usually involve the use of thermal and structural finite element methods (FEM) for which the utilisation of hundreds of thousands of finite elements is becoming common practice due to the computational power of modern computers. ${ }^{3}$ However, if a component is loaded cyclically and beyond the elastic limit of the material, phenomena such as isotropic and kinematic hardening, cyclic creep or relaxation can occur during operation and should be considered for both accurate stress-strain simulations and damage predictions. Analyses of thousands of cycles with changing material properties are unfortunately still too challenging, even for the high computational power of modern computers, therefore a stabilised state of the material is usually considered for analyses of components subjected to cyclic loadings which cause an elastoplastic material response. ${ }^{9,10}$ In a stabilised state, elastoplastic models including kinematic hardening of the material are usually considered whereas other cyclic phenomena are neglected. ${ }^{10}$ This enables analyses of stabilised loading histories only, and whilst still ensuring reliable thermomechanical damage predictions it considerably reduces computational time. In Fig. 1, a stabilised stress-strain response of ferritic stainless steel EN 1.4512 is depicted using an elastoplastic model with nonlinear kinematic hardening. Nonlinear kinematic hardening is usually the preferred choice as compared to bilinear kinematic hardening which oversimplifies the elastoplastic behaviour of the material. ${ }^{11}$ Furthermore, for metals with a symmetric stabilised stress-strain response in tension and compression, e.g. steel and aluminium alloys, it is sufficient to determine the stabilised cyclic curve of the plasticity model as the hysteresis branches can be simulated using Masing and memory rules. ${ }^{10}$ Every point on the cyclic curve represents a hysteresis loop with a tension and compression branch. A description of the cyclic curve of the material therefore also enables cyclic stress-strain

\footnotetext{
${ }^{1}$ University of Ljubljana, Faculty of Mechanical Engineering, Slovenia
}

\section{Corresponding author:}

Domen Šeruga, Faculty of Mechanical Engineering, Aškerčeva 6, SI1000 Ljubljana, Slovenia

Email: domen.seruga@fs.uni-lj.si 


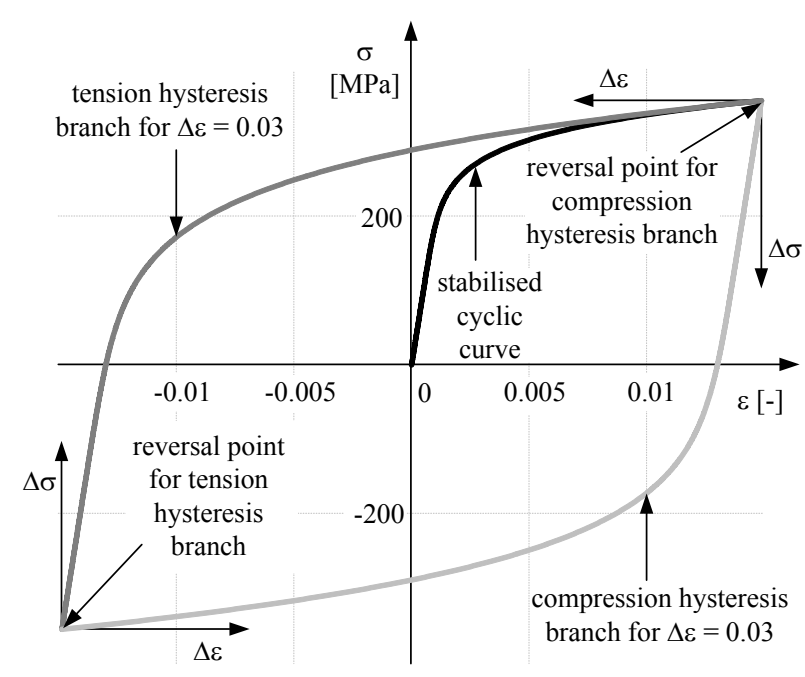

Figure 1. Stabilised stress-strain response at $20^{\circ} \mathrm{C}$ of ferritic stainless steel EN 1.4512 using an elastoplastic RO model with nonlinear kinematic hardening. Every point on the cyclic curve represents a hysteresis loop with a tension and compression branch (depicted for a hysteresis loop with $\Delta \varepsilon=0.03$ ).

modelling with kinematic hardening. A stabilised cyclic curve usually requires a parametric description of the stressstrain relation. ${ }^{12-14}$ This stress-strain relation is observed from experimental data gained by either low-cycle fatigue tests at several strain levels or incremental step tests. For damage predictions of thermomechanically loaded metals, it is often the case that a stabilised cyclic curve is described by the Ramberg-Osgood (RO) equation,

$$
\varepsilon=\frac{\sigma}{E}+\left(\frac{\sigma}{K^{\prime}}\right)^{\frac{1}{n^{\prime}}},
$$

where $E, K^{\prime}$ and $n^{\prime}$ represent elastic modulus, cyclic hardening coefficient and cyclic hardening exponent, respectively, $\sigma$ stands for the stress and $\varepsilon$ stands for the total strain. ${ }^{15,16}$ Combined by the Masing and memory rules, this material model can be used to describe the cyclic behaviour of metals with nonlinear kinematic hardening, ${ }^{10}$

$$
\Delta \varepsilon=\frac{\Delta \sigma}{E}+2\left(\frac{\Delta \sigma}{2 K^{\prime}}\right)^{\frac{1}{n^{\prime}}},
$$

where a new coordinate system $\Delta \sigma-\Delta \varepsilon$ must be inserted into each reversal point (Fig. 1). For many materials, RO material parameters have already been reported ${ }^{11,17-19}$ or they can be determined from raw experimental data. ${ }^{13,20-27}$ However, in finite element solvers, e.g. Abaqus, Eq. 1 is not available to describe the cyclic behaviour of the material during loading. In this case, a similar model can be used, i.e. the Armstrong-Frederick ( $\mathrm{AF}$ ) model, defined by

$$
\sigma=R_{0}+Q_{\infty}\left(1-e^{-\gamma \varepsilon_{\mathrm{p}}}\right)
$$

where $R_{0}$ represents the yield stress and $Q_{\infty}$ and $\gamma$ are kinematic hardening coefficients, $\varepsilon_{\mathrm{p}}$ stands for the plastic strain. ${ }^{1,12,28-32}$ Cyclic behaviour with nonlinear kinematic hardening in the case of AF model can be described as

$$
\Delta \sigma=2 R_{0}+2 Q_{\infty}\left(1-e^{-\gamma \frac{\Delta \varepsilon_{\mathrm{p}}}{2}}\right)
$$

where a new coordinate system $\Delta \sigma-\Delta \varepsilon$ must be inserted into each reversal point (Fig. 1). The stress-strain result of this model is similar but not equal because the RO model follows a power law, whereas the AF model is based on an exponential law as can be seen by comparing Eqs. 1 and 3 .

A material model based on Prandtl operators presented by Nagode et al. ${ }^{10}$ enables continuous modelling of elastoplastic cyclic material behaviour with nonlinear kinematic hardening where a variable thermal and mechanical load history exists and the determination of reversal points with Masing and memory rules becomes uncertain. ${ }^{33}$ It requires material parameters for the RO model at distinct test temperatures. Once these parameters have been obtained, such a model can then be used to simulate strain history from a known temperature and stress history of a variably thermomechanically loaded structural component. Some further advantages of this model are an extension to viscoplastic cyclic material behaviour, separate prediction of both fatigue and creep damage, and high computational speed. ${ }^{34,35}$ However, the temperature and stress histories must be exported from FEA where they are calculated according to the AF model if the RO model is not available.

It turns out that the material parameters of the $\mathrm{AF}$ model cannot be simply expressed by the RO material parameters. A numerical procedure must therefore be designed which enables the determination of those AF material parameters that will give a minimum least squares error between the original and the fitted model. The problem of determining the AF material parameters has already been addressed by various researchers. ${ }^{13,17,19-21,27,36}$ Hales et al. recommend best laboratory practice, test data analysis and the exploitation of existing cyclic stress-strain data. ${ }^{13}$ Wagner and Decker report on the simulation of thermomechanical deformation behaviour of a Nickel-base alloy. ${ }^{27}$ Zaletelj et al. deduced an equality between the RO and AF model in combination with a linear least squares fit to calculate the initial values of the AF model. ${ }^{19}$ Bromesson and Ekh developed an optimisation technique based on sequential use of the Downhill Simplex method followed by iterations with the gradient based Han-Powell method with Armijo line search until convergence. ${ }^{20} \mathrm{~A}$ study by Chaparro et al. separately compares methods on raw experimental data and combines methods in order to gain a final set of globally optimal values of material parameters. ${ }^{21}$ The majority of researchers report on the use of the LevenbergMarquardt algorithm to determine the parameters. ${ }^{21,27,36-38}$ Maciejewski et al. used a differential evolution method to fit the AF parameters of AISI 304 steel $^{25}$ and Franulovic et al. employed a genetic algorithm to determine the AF parameters for $42 \mathrm{CrMo} 4$ steel. ${ }^{39}$ Regardless of the method used, determining AF material parameters represents a multidimensional optimisation problem and the methods which are usually applied can reach a local minimum of the squared differences between the models, rather than a global minimum. ${ }^{21,40,41}$

For the titanium stabilised extra mild $12 \%$ chromium ferritic stainless steel EN 1.4512 used in the study, the AF material parameters need to be defined in order to perform FE simulations. The RO material parameters of the stabilised cyclic curves determined from low-cycle fatigue tests and the tensile material properties are only available at four 
temperatures: $20,300,650$ and $850{ }^{\circ} \mathrm{C}$ (raw experimental data are currently unavailable). ${ }^{3}$ These temperatures were chosen so as to cover the expected temperature range of the components during thermal shock tests. ${ }^{9}$ As the most suitable optimisation method for the determination of the AF material parameters for the material under investigation has not yet been reported in the available literature, the main objective was to investigate whether any of the optimisation methods has distinctive advantages or disadvantages. In order to achieve this, the four most commonly used optimisation methods were chosen to address the material parameter determination of the exponential law (Eq. 3), whilst knowing in advance the material parameters of the power law (Eq. 1). These methods are the Gauss-Newton (GN) method, the Levenberg-Marquardt (LM) method, the Downhill Simplex (DS) method also known as the NelderMead method and a genetic algorithm (GA). The GN and the LM methods represent searching with derivatives of functions whereas the DS and the GA methods represent direct search algorithms without the necessity of knowing the partial derivatives of the functions in advance. The GN, LM and DS methods will produce different local optima given a different initial search position, whereas the GA method will give a global optimum resulting from a variety of initial search positions. In order to gain the global optimum for the comparison regardless of the method used for optimisation of material parameters, an algorithm is designed which also allows setting an arbitrary number of searches with the GN, LM and DS methods. The optimal values of the AF material parameters for the ferritic stainless steel EN 1.4512 were then tested to determine how they vary depending on which optimisation method is applied.

\section{Methods}

An arbitrary number of points $\left(\sigma_{i}, \varepsilon_{i}\right) ; i=1, \ldots, n$ is generated between the estimated yield stress $R_{0 \mathrm{e}}$ and the tensile strength $R_{\mathrm{m}}$ from prior knowledge of the RO material parameters. These points are uniformly distributed between the total yield energy $W_{1}$ and the total tensile energy $W_{\mathrm{n}}$, calculated as

$$
\begin{aligned}
& W_{1}=R_{0 \mathrm{e}} \cdot \varepsilon\left(R_{0 \mathrm{e}}\right) \\
& W_{\mathrm{n}}=R_{\mathrm{m}} \cdot \varepsilon\left(R_{\mathrm{m}}\right)
\end{aligned}
$$

The distribution of points between $W_{1}$ and $W_{\mathrm{n}}$ given in Eq. 5 ensures the total energy in the material in the plastic area is represented equally during the search of optimal values of the AF material parameters (Fig. 2). The estimated yield stress of the material $R_{0 \mathrm{e}}$ can be established e.g. by the strain of $\varepsilon=0.002$ at which $0.2 \%$ of permanent strain remains in the material after unloading ${ }^{38}$ whereas the optimal yield stress $R_{0}$ and parameters $Q_{\infty}$ and $\gamma$ are obtained later on by minimisation of the function $R^{2}$.

The error between the RO and the AF models is calculated as the sum of squared differences over $n$ chosen points (Fig. 2). Hence an objective function $R^{2}$ can be introduced as

$$
R^{2}=\sum_{i=1}^{\mathrm{n}}\left(\sigma_{i}-R_{0}-Q_{\infty}\left(1-e^{-\gamma \varepsilon_{\mathrm{p} i}}\right)\right)^{2}
$$

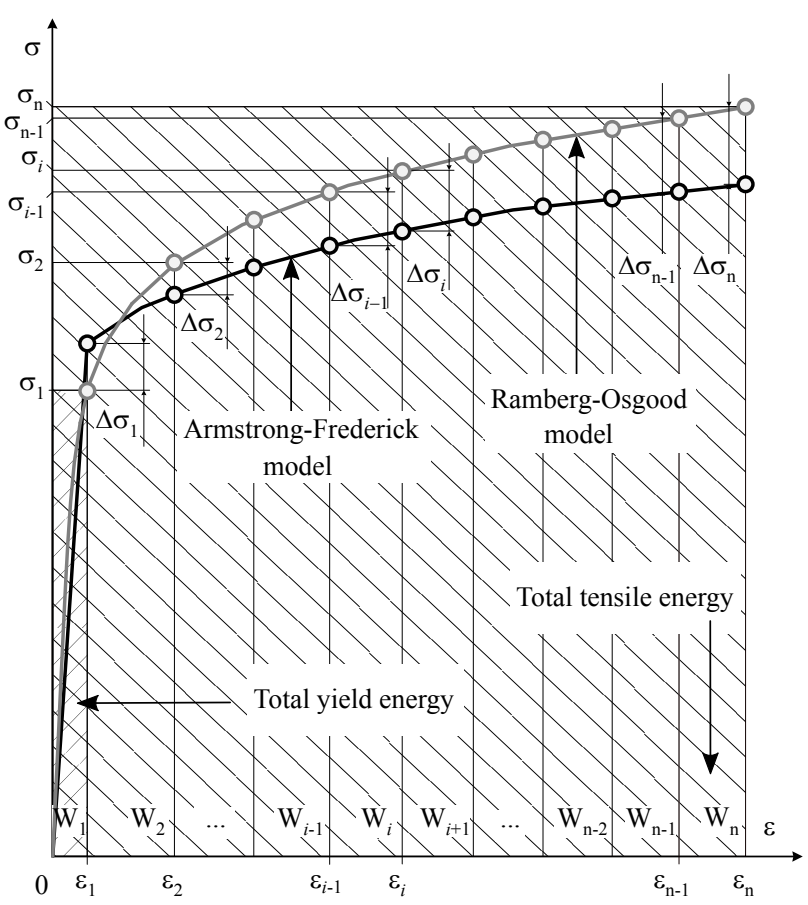

Figure 2. Points are uniformly distributed in the plastic area between the estimated total yield energy and the total tensile energy. A minimum error between the $\mathrm{RO}$ and the $\mathrm{AF}$ model is searched for as a sum of squared differences over the chosen points.

Optimisation methods are now run separately for the determination of the AF material parameters to obtain the method-dependent global minima of the objective functions $\min \left(R_{\mathrm{gn}}^{2}\right)$, $\min \left(R_{\mathrm{lm}}^{2}\right)$, $\min \left(R_{\mathrm{ds}}^{2}\right)$ and $\min \left(R_{\mathrm{ga}}^{2}\right)$. The vectors $\mathbf{x}_{\mathrm{gn}}^{\mathrm{opt}}=\left\{\begin{array}{c}R_{0} \\ Q_{\infty} \\ \gamma\end{array}\right\}_{\mathrm{gn}}^{\mathrm{opt}}, \mathbf{x}_{\mathrm{lm}}^{\mathrm{opt}}=\left\{\begin{array}{c}R_{0} \\ Q_{\infty} \\ \gamma\end{array}\right\}_{\mathrm{lm}}^{\mathrm{opt}}, \mathbf{x}_{\mathrm{ds}}^{\mathrm{opt}}=$ $\left\{\begin{array}{c}R_{0} \\ Q_{\infty} \\ \gamma\end{array}\right\}_{\mathrm{ds}}^{\mathrm{opt}}$ and $\mathbf{x}_{\mathrm{gat}}^{\mathrm{opt}}=\left\{\begin{array}{c}R_{0} \\ Q_{\infty} \\ \gamma\end{array}\right\}_{\mathrm{ga}}^{\mathrm{opt}}$ will contain the method-dependent final sets of optimal values of material parameters, each determined by

$$
\mathbf{x}^{\mathrm{opt}}=\operatorname{argmin}\left(R^{2}\right) .
$$

Since the optimisation methods used in the study are generally known, only a brief description is outlined here and the method-specific quantities are defined for the problem of determining the AF material parameters. Further details of the methods can be found in other sources. ${ }^{42-53}$

\section{Damped Gauss-Newton method}

In order to find the optimal values of the parameters, the minimum of the objective function in Eq. 6 is searched for,

$$
\min \left(R^{2}\right)=\min \left(\sum_{i=1}^{\mathrm{n}}\left(\sigma_{i}-R_{0}-Q_{\infty}\left(1-e^{-\gamma \varepsilon_{\mathrm{p} i}}\right)\right)^{2}\right)
$$

by changing parameters $R_{0}, \gamma$ and $Q_{\infty}$. From $m$ initial trial sets of solutions $\mathbf{x}_{\text {gn }}^{j} \sim \mathcal{U}\left(\mathbb{R}^{+}\right) ; j=1, \ldots, m$, new increments $\Delta \mathbf{x}_{\mathrm{gn}}^{j} ; j=1, \ldots, m$ in the optimal directions are 
calculated as

$$
\Delta \mathbf{x}_{\mathrm{gn}}^{j}=\beta \mathbf{H}^{-1} \mathbf{J}
$$

The iterative search of the optimal solution hence requires the calculation of both the Jacobian of the objective function,

$$
\begin{aligned}
\mathbf{J} & =\frac{\partial R^{2}}{\partial \mathbf{x}_{\mathrm{gn} k}^{j}}=\left\{\begin{array}{c}
\frac{\partial R^{2}}{\partial Q_{\infty}^{j}} \\
\frac{\partial R^{2}}{\partial \gamma^{j}} \\
\frac{\partial R^{2}}{\partial R_{0}^{j}}
\end{array}\right\}= \\
& =-2 \sum_{i=1}^{\mathrm{n}}\left\{\begin{array}{c}
\left(\begin{array}{c}
\left.\sigma_{i}-R_{0}^{j}-Q_{\infty}^{j} A_{i}^{j}\right) A_{i}^{j} \\
\left(\sigma_{i}-R_{0}^{j}-Q_{\infty}^{j} A_{i}^{j}\right) B_{i}^{j} \\
\left(\sigma_{i}-R_{0}^{j}-Q_{\infty}^{j} A_{i}^{j}\right)
\end{array}\right\} ; k=1,2,3
\end{array}\right.
\end{aligned}
$$

and the Hessian of the objective function,

$$
\begin{aligned}
\mathbf{H} & =\frac{\partial^{2} R^{2}}{\partial \mathbf{x}_{\mathrm{gn} k}^{j} \partial \mathbf{x}_{\mathrm{gn} l}^{j}}=\left[\begin{array}{ccc}
\frac{\partial^{2} R^{2}}{\partial Q_{\infty}^{j} \partial Q_{\infty}^{j}} & \frac{\partial^{2} R^{2}}{\partial Q_{\infty}^{j} \partial \gamma^{j}} & \frac{\partial^{2} R^{2}}{\partial Q_{\infty}^{j} \partial R_{0}^{j}} \\
\frac{\partial^{2} R^{2}}{\partial \gamma^{j} \partial Q_{\infty}^{j}} & \frac{\partial^{2} R^{2}}{\partial \gamma^{j} \partial \gamma^{j}} & \frac{\partial^{2} R^{2}}{\partial \gamma^{j} \partial R_{0}^{j}} \\
\frac{\partial^{2} R^{2}}{\partial R_{0}^{j} \partial Q_{\infty}^{j}} & \frac{\partial^{2} R^{2}}{\partial R_{0}^{j} \partial \gamma^{j}} & \frac{\partial^{2} R^{2}}{\partial R_{0}^{j} \partial R_{0}^{j}}
\end{array}\right] \\
& =2 \sum_{i=1}^{\mathrm{n}}\left[\begin{array}{ccc}
\left(A_{i}^{j}\right)^{2} & A_{i}^{j} B_{i}^{j} & A_{i}^{j} \\
A_{i}^{j} B_{i}^{j} & \left(B_{i}^{j}\right)^{2} & B_{i}^{j} \\
A_{i}^{j} & B_{i}^{j} & 1
\end{array}\right] ; k, l=1,2,3
\end{aligned}
$$

where $A_{i}^{j}=1-e^{-\gamma^{j} \varepsilon_{\mathrm{p} i}}$ and $B_{i}^{j}=Q_{\infty}^{j} \varepsilon_{\mathrm{p} i} e^{-\gamma^{j} \varepsilon_{\mathrm{p} i}}$. The terms of the Hessian including the second derivatives of Eq. 3 are omitted for stability. ${ }^{42,44,53}$ The coefficient $\beta$ is set to a small constant value which ensures a stable approach to the optimal solution.

\section{Levenberg-Marquardt method}

For the same objective function and Jacobian as given in Eqs. 8 and 10 and for $m$ initial trial sets of solutions $\mathbf{x}_{\operatorname{lm}}^{j} \sim$ $\mathcal{U}\left(\mathbb{R}^{+}\right) ; j=1, \ldots, m$, new increments $\Delta \mathbf{x}_{\mathrm{lm}}^{j} ; j=1, \ldots, m$ in the optimal directions are calculated as

$$
\Delta \mathbf{x}_{\operatorname{lm}}^{j}=\beta \mathbf{H}^{*-1} \mathbf{J}
$$

where $\mathbf{H}^{*}$ represents the modified Hessian of the objective function according to Levenberg and Marquardt ${ }^{48,50,53}$ with diagonal elements multiplied by a dynamic constant $\lambda$, which regulates the rate of approach to the optimal solution,

$$
\begin{aligned}
& \begin{aligned}
\mathbf{H}^{*}= & \frac{\partial^{2} R^{2}}{\partial \mathbf{x}_{\operatorname{lm} k}^{j} \partial \mathbf{x}_{\operatorname{lm} l}^{j}} \cdot\left\{\begin{array}{cc}
(1+\lambda) & \text { if } k=l \\
1 & \text { otherwise }
\end{array}=\right. \\
= & {\left[\begin{array}{ccc}
\frac{\partial^{2} R^{2}}{\partial Q_{\infty}^{j} \partial Q_{\infty}^{j}} & \frac{\partial^{2} R^{2}}{\partial Q_{\infty}^{j} \partial \gamma^{j}} & \frac{\partial^{2} R^{2}}{\partial Q_{\infty}^{j} \partial R_{0}^{j}} \\
\frac{\partial^{2} R^{2}}{\partial \gamma^{j} \partial Q_{\infty}^{j}} & \frac{\partial^{2} R^{2}}{\partial \gamma^{j} \partial \gamma^{j}} & \frac{\partial^{2} R^{2}}{\partial \gamma^{j} \partial R_{0}^{j}} \\
\frac{\partial^{2} R^{2}}{\partial R_{0}^{j} \partial Q_{\infty}^{j}} & \frac{\partial^{2} R^{2}}{\partial R_{0}^{j} \partial \gamma^{j}} & \frac{\partial^{2} R^{2}}{\partial R_{0}^{j} \partial R_{0}^{j}}
\end{array}\right] . } \\
& \left\{\begin{array}{llll}
(1+\lambda) & \text { if } k=l \\
1 & \text { otherwise }
\end{array}\right. \\
= & 2 \sum_{i=1}^{\mathrm{n}}\left[\begin{array}{ccc}
\left(A_{i}^{j}\right)^{2}(1+\lambda) & A_{i}^{j} B_{i}^{j} & A_{i}^{j} \\
A_{i}^{j} B_{i}^{j} & \left(B_{i}^{j}\right)^{2}(1+\lambda) & B_{i}^{j} \\
A_{i}^{j} & B_{i}^{j} & (1+\lambda)
\end{array}\right] ;
\end{aligned} \\
& k, l=1,2,3
\end{aligned}
$$

where $A_{i}^{j}=1-e^{-\gamma^{j} \varepsilon_{\mathrm{p} i}}$ and $B_{i}^{j}=Q_{\infty}^{j} \varepsilon_{\mathrm{p} i} e^{-\gamma^{j} \varepsilon_{\mathrm{p} i}}$. If the objective function in the current step is smaller than the objective function in the previous step, the dynamic constant $\lambda$ is increased. Conversely, if the objective function in the current step is greater than the objective function in the previous step, the dynamic constant $\lambda$ is decreased.

\section{Downhill Simplex method}

For $p$ unknown material parameters, $m$ simplexes with $p+1$ nodes are generated with initial values of the objective functions in the nodes of the simplexes as 51,53

$$
\begin{aligned}
y_{k}^{j} & =\sum_{i=1}^{\mathrm{n}}\left(\sigma_{i}-R_{0}^{j}-Q_{\infty, k}^{j}\left(1-e^{-\gamma_{k}^{j} \varepsilon_{\mathrm{p} i}}\right)\right)^{2} \\
j & =1, \ldots, m ; k=1, \ldots, p+1
\end{aligned}
$$

where $j$ and $k$ represent the number of the simplex and the number of the node, respectively. Each node $P_{k}^{j}$ represents a vector of material parameters $\mathbf{x}_{\mathrm{ds}, k}^{j}$ and the initial values of $P_{k}^{j}$ are uniformly distributed, $P_{k}^{j} \sim \mathcal{U}\left(\mathbb{R}^{+}\right)$. The node with the highest value of the objective function is denoted as $P_{\mathrm{h}}^{j}$, the node with the second highest value of the objective function is denoted as $P_{\mathrm{nh}}^{j}$ and the node with the lowest value of the objective function is denoted as $P_{1}^{j}$. In order to find the minimum of the objective function, three operations are performed. First, a reflection of $P_{\mathrm{h}}^{j}$ is tried as

$$
P_{*}^{j}=\lambda P_{\mathrm{h}}^{j}+(1-\lambda) \bar{P}^{j} ; j=1, \ldots, m
$$

where $\bar{P}^{j}$ stands for the centroid of the nodes $P_{k}^{j} \neq P_{\mathrm{h}}^{j} ; k=$ $1, \ldots, p+1$ of the $j$-th simplex. The coefficient $\lambda$ is set to the value of -1 . If $y_{*}^{j}>y_{\mathrm{h}}^{j}$, then $P_{\mathrm{h}}^{j}$ remains as it was, otherwise $P_{\mathrm{h}}^{j}=P_{*}^{j}$ and a new simplex is generated. Second, if $y_{*}^{j}<y_{1}^{j}$, an expansion of the simplex is tried as

$$
P_{* *}^{j}=\lambda P_{*}^{j}+(1-\lambda) \bar{P}_{*}^{j} ; j=1, \ldots, m
$$

where $\bar{P}_{*}^{j}$ stands for the centroid of the nodes $P_{k}^{j} \neq P_{*}^{j} ; k=$ $1, \ldots, p+1$ of the $j$-th simplex. The coefficient $\lambda$ is now set to the value of 2 . If $y_{* *}^{j}<y_{1}^{j}$, the node with the highest value of the objective function is replaced by $P_{\mathrm{h}}^{j}=P_{* *}^{j}$, a new simplex is generated and the procedure of finding the minimum of the objective function is repeated. Third, if $y_{*}^{j}>y_{\mathrm{nh}}^{j}$, a contraction of the simplex is tried with Eq. 16 where the coefficient $\lambda$ is set to the value of 0.5 . If $y_{* *}^{j}<y_{\mathrm{h}}^{j}$, the node with the highest value of the objective function is replaced by $P_{\mathrm{h}}^{j}=P_{* *}^{j}$, a new simplex is generated and the procedure of finding a better solution is repeated. If $y_{* *}^{j} \geq$ $y_{\mathrm{h}}^{j}$, a multiple contraction of the simplex around the node with the lowest value of the objective function is performed as

$$
P_{k}^{j}=\frac{P_{k}^{j}+P_{1}^{j}}{2} ; j=1, \ldots, m ; k=1, \ldots, p+1
$$

and the procedure of finding a better solution is repeated. The final set of optimal values of material parameters is written in the vector $\mathbf{x}_{\mathrm{ds}}^{\mathrm{opt}}=\min \left(P_{1}^{j}\right) j=1, \ldots, m$ when the convergence criterion for each simplex has been reached.

\section{Genetic algorithm}

In order to find the set of optimal values of material parameters $\mathbf{x}_{\text {ga }}{ }^{\text {opt }}$ by the genetic algorithm, $p$ generations, each consisting of $m$ chromosomes, are utilised to carry out the search. An initial population is uniformly distributed as $C_{1}^{j} \longleftarrow \mathbf{x}_{\text {ga }}^{j} \sim \mathcal{U}\left(\mathbb{R}^{+}\right) ; j=1, \ldots, m$ and the 
$k$-th population is denoted as $C_{k}^{j} ; k=1, \ldots, p$. Every chromosome $C_{k}^{j}$ contains $2 q$ genes where $q$ stands for the number of unknown material parameters, each consisting of two parts, integer and decimal. Every decimal gene $g_{10}$ is furthermore converted into a binary form $g_{2}$ as

$$
\begin{aligned}
g_{10} & =a_{\mathrm{n}-1} \cdot 2^{\mathrm{n}-1}+a_{\mathrm{n}-2} \cdot 2^{\mathrm{n}-2}+\cdots \\
& +a_{1} \cdot 2^{1}+a_{0} \cdot 2^{0}
\end{aligned}
$$

where $a_{\mathrm{n}-1}, a_{\mathrm{n}-2}, \ldots, a_{1}, a_{0}$ represent bits in gene $g_{2}$ and $n$ stands for the number of bits in this gene. The objective function in Eq. 6 is evaluated for every chromosome in the $k$-th population and the fitness of the chromosomes $\alpha_{k}^{j}$ is calculated as

$$
\alpha_{k}^{j}=\frac{\frac{1}{1+R_{j}^{2}}}{\sum_{j=1}^{\mathrm{m}} \frac{1}{1+R_{j}^{2}}} ; j=1, \ldots, m
$$

Selection of those chromosomes suitable as candidates for reproduction $R_{k}^{j}$ is performed by the roulette selection for which the cumulative probabilities of fitness values $\beta_{k}^{j}$ are determined as

$$
\beta_{k}^{j}=\sum_{i=1}^{j} \frac{1}{1+R_{i}^{2}} ; j=1, \ldots, m .
$$

Roulette selection generates random values $x_{k}^{j} \sim$ $\mathcal{U}(0,1) ; j=1, \ldots, m$ and the reproduction is carried out as

$$
R_{k}^{j}=C_{k}^{i} ; i=\max \left(l \mid\left(x_{k}^{j} \leq \beta_{k}^{l}\right)\right) ; j, l=1, \ldots, m .
$$

Next, first parental chromosomes $P_{1 k}^{i}$ are chosen among chromosomes $R_{k}^{j}$ as

$$
\begin{aligned}
& P_{1 k}^{i}=R_{k}^{j} \mid\left(y_{k}^{j}<C_{\mathrm{co}}\right) ; j=1, \ldots, m ; i=1, \ldots, r \\
& p_{k}^{i}=j ; j=1, \ldots, m ; i=1, \ldots, r
\end{aligned}
$$

where the selection is based on prior random values $y_{k}^{j} \sim$ $\mathcal{U}(0,1) ; j=1, \ldots, m$ and $C_{\text {co }}$ is a constant, representing the crossover rate. The number of parents is represented by $r$ and the position of the first parent in the $k$-th generation is marked with $p_{k}^{i}$. The second parental chromosomes $P_{2 k}^{i}$ are determined as

$$
P_{2 k}^{i}=P_{1 k}^{\lfloor j\rfloor} \mid(\lfloor j\rfloor \neq i) ; j \sim \mathcal{U}(1, r) ; i=1, \ldots, r
$$

and the position where a pair of parental chromosomes will exchange their genes is decided as

$$
b_{k}^{i}=\lfloor y\rfloor_{k}^{i} ; y_{k}^{i} \sim \mathcal{U}(1,2 q \cdot n) ; i=1, \ldots, r
$$

where $\lfloor\cdot\rfloor$ represents the floor function and $2 q \cdot n$ is the total length of the bits in a parental chromosome. A single-point crossover is chosen between two parents. Chromosomes of the offspring generation $C_{k+1}^{j}$ are thus gained either by reproduction or crossover as

$$
\begin{gathered}
C_{k+1}^{j}\left\{\begin{array}{cr}
=R_{k}^{j} & \text { if } j \neq p_{k}^{l} ; \\
\subset\left(P_{1 k}^{l} \mid\left(i \leq b_{k}^{l}\right)\right) \wedge\left(P_{2 k}^{l} \mid\left(i>b_{k}^{l}\right)\right) & \text { otherwise; }
\end{array}\right. \\
i=1, \ldots, 2 q \cdot n ; j=1, \ldots, m ; l=1, \ldots, r .
\end{gathered}
$$

Mutations can also appear in the next generation of the chromosomes. The total number of mutations $C_{\text {mut }}$ is first determined as

$$
C_{\mathrm{mut}}=c_{\mathrm{mut}} \cdot 2 q \cdot n \cdot m
$$

where $c_{\text {mut }}$ and $2 q \cdot n \cdot m$ represent the mutation rate and the total number of bits in the population, respectively. The chromosomes of the offspring generation are concatenated into a single string representing the population. The places of mutations in the population $d_{k+1}^{i}$ are determined as

$$
\begin{aligned}
d_{k+1}^{i} & =\lfloor z\rfloor_{k+1}^{i} ; z_{k+1}^{i} \sim \mathcal{U}(1,2 q \cdot n \cdot m) \\
i & =1, \ldots, C_{\mathrm{mut}}
\end{aligned}
$$

and the bits in the population are swapped at these places. The final set of optimal values of material parameters is written in the vector $\mathbf{x}_{\mathrm{ga}}^{\mathrm{opt}}=C_{p}^{i} \mid\left(i=\underset{j=1, \ldots, m}{\operatorname{argmax}}\left(\alpha_{\mathrm{p}}^{j}\right)\right)$ when the pre-set number of generations $p$ has been reached.

\section{Comparison}

The methods have been compared for the material under investigation given the tensile properties and the RO material parameters at four experimental temperatures of 20, 300, 650 and $850{ }^{\circ} \mathrm{C}$ (Table 1). For these temperatures, AF material parameters have been calculated by the GN, LM, DS and GA methods. They are given in Table 2 and the associated cyclic curves are presented in Fig. 3. Four quantities have been followed throughout the optimisation process to evaluate the methods: values of AF material parameters, the $R^{2}$ value, the number of steps to reach the optimal set of AF material parameters and the processing time of the methods. $\mathrm{R}$ language has been used to carry out the calculations. One hundred points on the cyclic curve were created to carry out the optimisation. For the GN, LM and DS methods, 100 independent parallel searches have been chosen and for the GA method, 10000 generations have been chosen, each with 100 chromosomes, crossover rate of 0.20 and mutation rate of 0.02 . The initial search area was chosen between 0 and $R_{0 \mathrm{e}}$ for parameter $R_{0}$, between 0 and $1000 \mathrm{MPa}$ for parameter $Q_{\infty}$ and between 0 and 1000 for parameter $\gamma$ regardless of the method, although optimal values of the material parameters were not bounded by these values. The coefficient $\beta$ for searching with the GN method was set to 0.1 and the dynamic constant $\lambda$ of the LM method was set to 0.001 and could be multiplied by factors of 2 or 0.5 during the search, depending on the convergence rate. The $R^{2}$ values per iteration for $20{ }^{\circ} \mathrm{C}$ are graphically presented in Fig. 4. The number of steps $N$ for each method to reach the optimal values of the material parameters is given in Fig. 5 and the processing time per search $t_{\mathrm{s}}$ is given in Fig. 6 . For easier comparisons in Figs. 5 and 6, normal distributions have been fitted to $N$ and $t_{\mathrm{s}}$.

\section{Discussion}

The global optima or the vicinity of the globally optimal values of the AF material parameters can be always achieved by all the methods compared here. Though the values of the material parameters given in Table 2 differ slightly, the cyclic 
Table 1. Values of the RO material parameters of the stabilised cyclic curves and tensile strength for EN 1.4512 steel used in this study.

\begin{tabular}{lllll}
\hline$T\left[{ }^{\circ} \mathrm{C}\right]$ & $E[\mathrm{MPa}]$ & $K^{\prime}[\mathrm{MPa}]$ & $n^{\prime}[-]$ & $R_{\mathrm{m}}[\mathrm{MPa}]$ \\
\hline 20 & 200000 & 603.42 & 0.1211 & 407 \\
300 & 180000 & 508.16 & 0.1103 & 360 \\
650 & 150000 & 183.13 & 0.0336 & 165 \\
850 & 110000 & 101.317 & 0.0052 & 99 \\
\hline
\end{tabular}
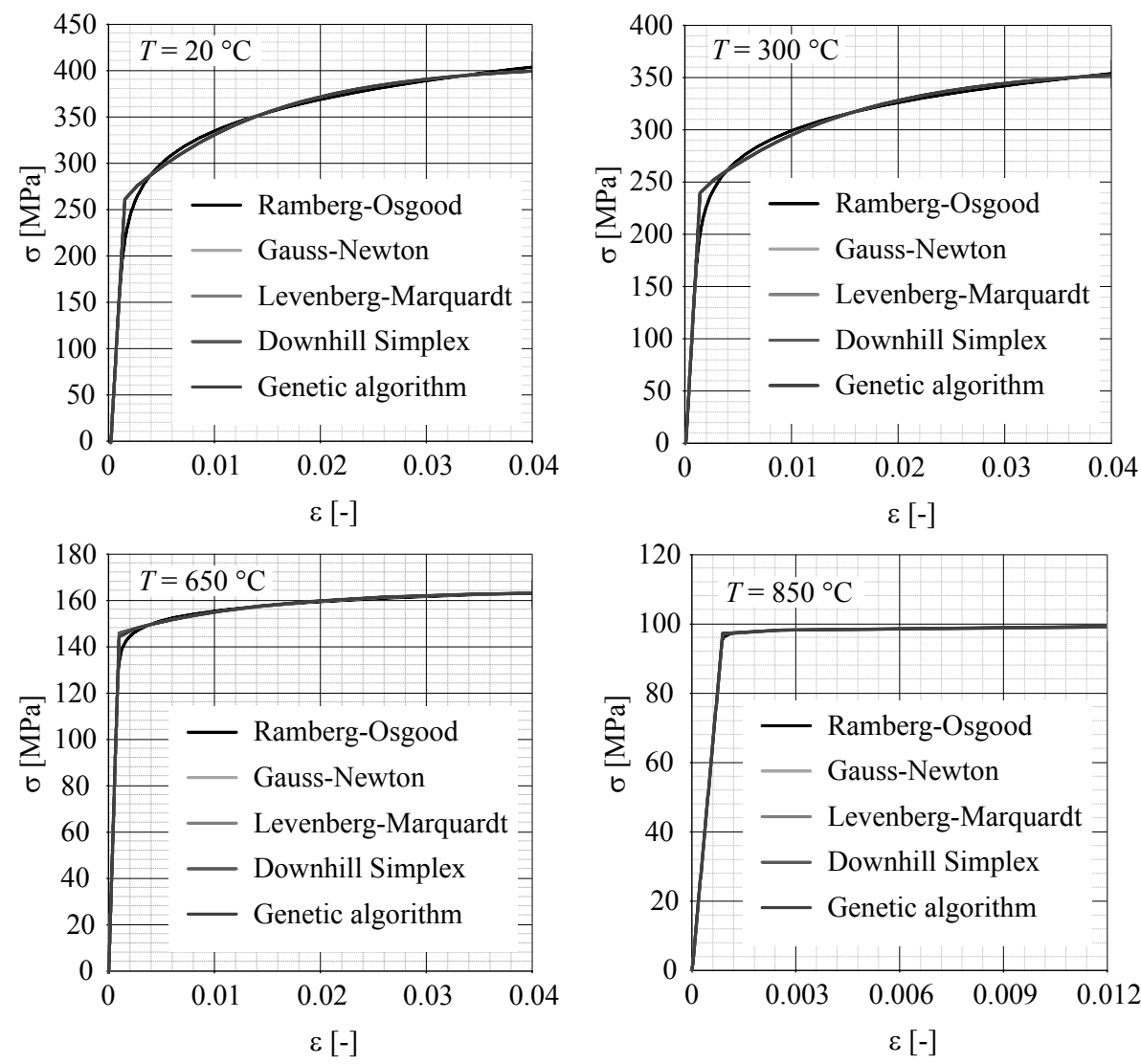

Figure 3. Comparison of temperature dependent cyclic curves described by the RO parameters and the AF parameters gained by different methods.

curves in Fig. 3 show no difference between strain values of 0 and 0.04 for temperatures of 20,300 and $650{ }^{\circ} \mathrm{C}$ and between 0 and 0.012 for $850{ }^{\circ} \mathrm{C}$. It can be noticed that for the material studied here, the cyclic curve at $850{ }^{\circ} \mathrm{C}$ differs from the cyclic curves at lower experimental temperatures, i.e. after reaching the yield stress at $850^{\circ} \mathrm{C}$, the stress barely increases with increasing strain (Fig. 3). The reason for this shape of the cyclic curve is the proximity of the recrystallisation temperature where hardening behaviour of the material is reducing.

The vast majority of searches using one of the GN, LM or DS methods does converge to the global optimum, however it is possible that occasionally a search does not (as can be seen in Fig. 4 for the DS method by the green circle). The reason for non-optimal final values of material parameters is most probably the convergence criterion which has been set equally for all the methods as $0.1 \%$ for the difference of the objective functions between two successive steps during a search. If the value of the objective function is already low and the next calculated step is small, the convergence criterion will be fulfilled although the global optimum has not yet been reached for that particular search. As this is the case only exceptionally, the globally optimal values are usually reached. This can be seen by comparing the values of the material parameters in Table 2, where there are practically no differences between the final values gained by the LM, DS and GN methods. The GN method however always slightly differs from the former two which is again the consequence of the convergence criterion. On the contrary, the GA method differs the most from the optimal values gained by other methods for all the temperatures and especially for $850{ }^{\circ} \mathrm{C}$ (Table 2). The reason for this behaviour lies in the principle of the search using the GA method. As only the number of generations is set in advance, there is no convergence criterion with the GA method. The crossover rate and the mutation rate define how quickly and how stable the GA method will approach the final set of material parameters. The latter is also responsible for a continuous deviation around the current solution and therefore for the largest discrepancy of the optimal values as 

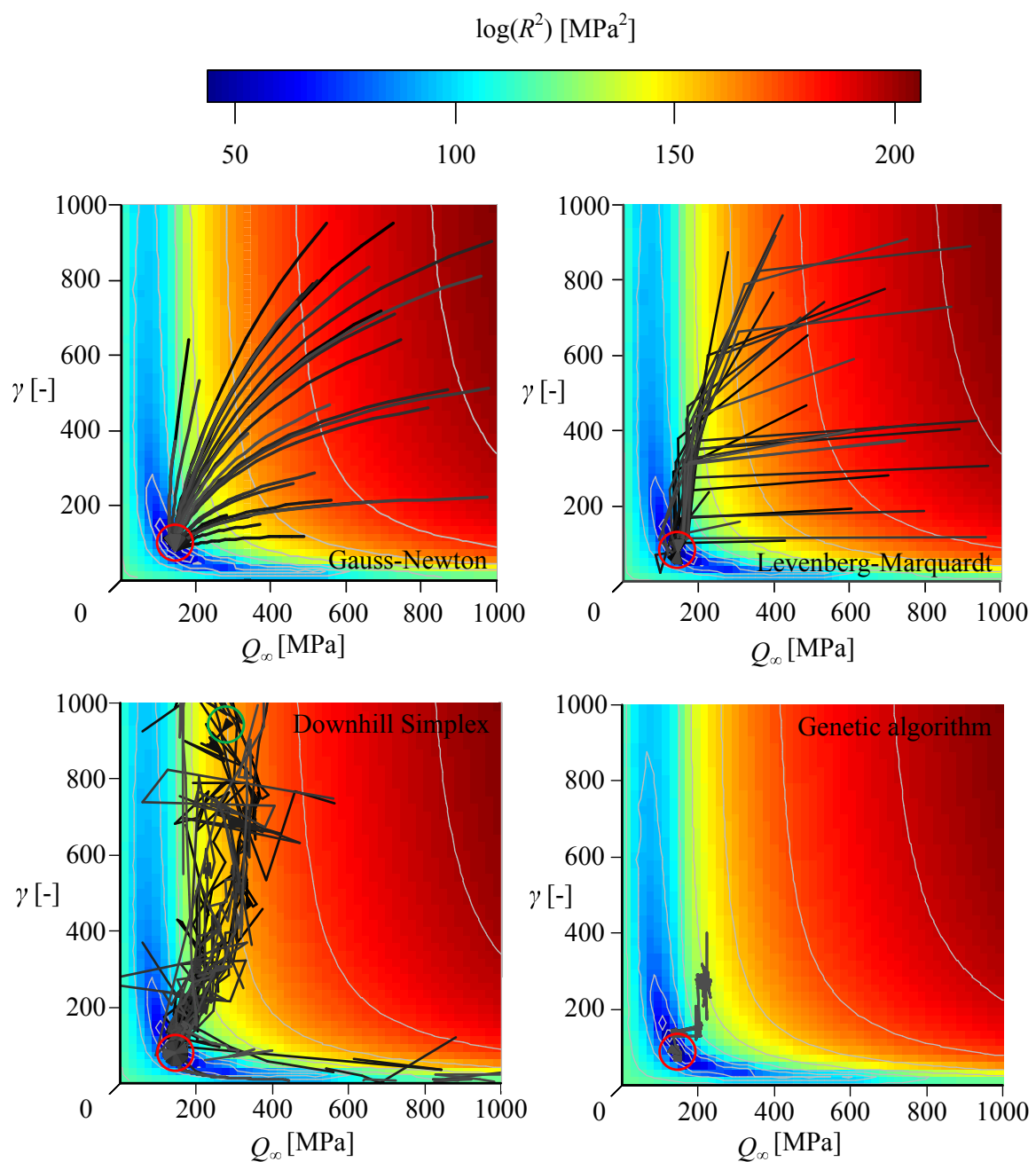

Figure 4. Comparison of 30 search paths projected onto the plane of the optimal value of $R_{0}$ as given in Table 2 for the GN, LM, DS and GA methods at a temperature of $20^{\circ} \mathrm{C}$ (from top left to bottom right). Red circles show the optimal values of material parameters $Q_{\infty}$ and $\gamma$.

Table 2. Optimal values of the AF material parameters for EN 1.4512 steel calculated in this study.

\begin{tabular}{llllllllllllll}
\hline Method & \multicolumn{3}{c}{ Gauss-Newton } & \multicolumn{3}{c}{ Levenberg-Marquardt } & \multicolumn{3}{c}{ Downhill Simplex } & \multicolumn{3}{c}{ Genetic algorithm } \\
\hline$T\left[{ }^{\circ} \mathrm{C}\right]$ & $R_{0}[\mathrm{MPa}]$ & $Q_{\infty}[\mathrm{MPa}]$ & $\gamma[-]$ & $R_{0}[\mathrm{MPa}]$ & $Q_{\infty}[\mathrm{MPa}]$ & $\gamma[-]$ & $R_{0}[\mathrm{MPa}]$ & $Q_{\infty}[\mathrm{MPa}]$ & $\gamma[-]$ & $R_{0}[\mathrm{MPa}]$ & $Q_{\infty}[\mathrm{MPa}]$ & $\gamma[-]$ \\
\hline 20 & 265.20 & 143.79 & 78.58 & 265.42 & 143.58 & 78.03 & 265.43 & 143.58 & 78.01 & 265.13 & 144.14 & 77.81 \\
300 & 242.38 & 118.41 & 72.06 & 242.55 & 118.15 & 71.66 & 242.55 & 118.15 & 71.67 & 241.73 & 118.89 & 72.41 \\
650 & 146.80 & 18.14 & 76.20 & 146.82 & 18.11 & 75.85 & 146.81 & 18.11 & 75.88 & 146.41 & 18.34 & 80.85 \\
850 & 97.54 & 1.56 & 216.11 & 97.54 & 1.56 & 215.85 & 97.54 & 1.56 & 215.90 & 97.69 & 2.00 & 106.90 \\
\hline
\end{tabular}

compared to the other methods. Though the final values of the material parameters can vary depending on the method, a comparison of the cyclic curves in Fig. 3 shows very little or practically no deviation between the cyclic curves in the fitted stress region between $R_{0 \mathrm{e}}$ and $R_{\mathrm{m}}$. Due to the difference between the models (exponential AF and power RO model), a discrepancy between the original and the fitted curve is however always present. It is especially noticeable around a yield strain of 0.002 in Fig. 3. This difference can be reduced by changing the parameter $R_{0}$. The change can be made prior to searching by setting $R_{0}$ to a constant value that corresponds to a pre-set value of strain, e.g. to 0.0001 or to 0.002 as shown in Fig. 7 for $20^{\circ} \mathrm{C}$. It turns out however, that with a three-parameter optimisation, the difference between the RO and AF curves is kept to a minimum over the whole range, whereas setting $R_{0}$ to other values reduces the difference between the cyclic curves around the yield stress. This is the case when $R_{0}$ is set to the constant value that corresponds to a strain of 0.002 , whilst the difference in the other regions between the curves increases.

The differences between the numbers of steps to reach the optimal set of material parameters and the processing times per search are significant between the methods (Figs. 5 and 6). On average, the LM method ensures the smallest number of steps to reach the convergence as can be seen by following the $R^{2}$ value in successive steps (Fig. 8), furthermore it requires the shortest time to achieve convergence. With the changing shapes of the curves however, the computational 

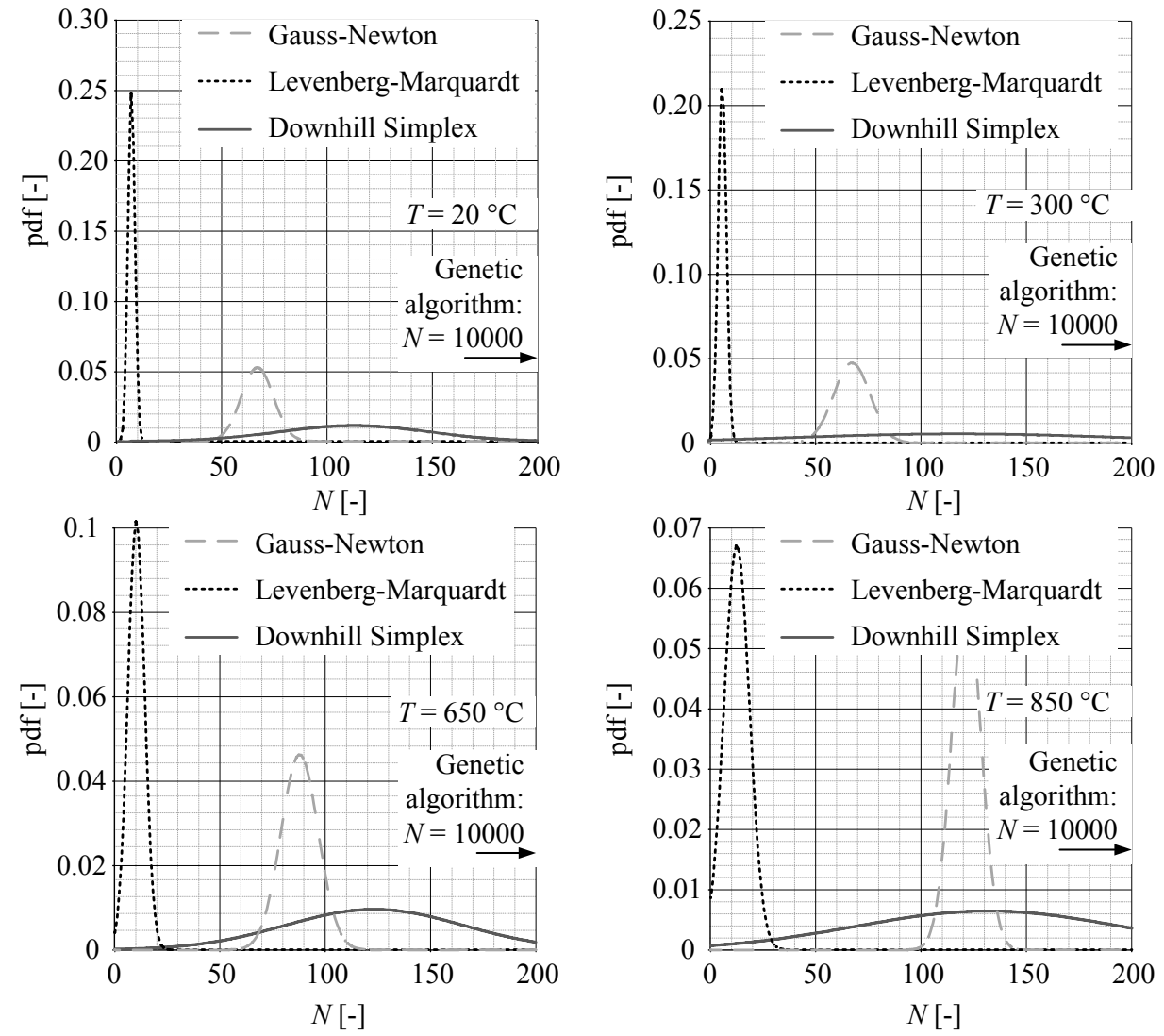

Figure 5. Number of steps to reach the optimal set of material parameters $R_{0}, Q_{\infty}$ and $\gamma$.
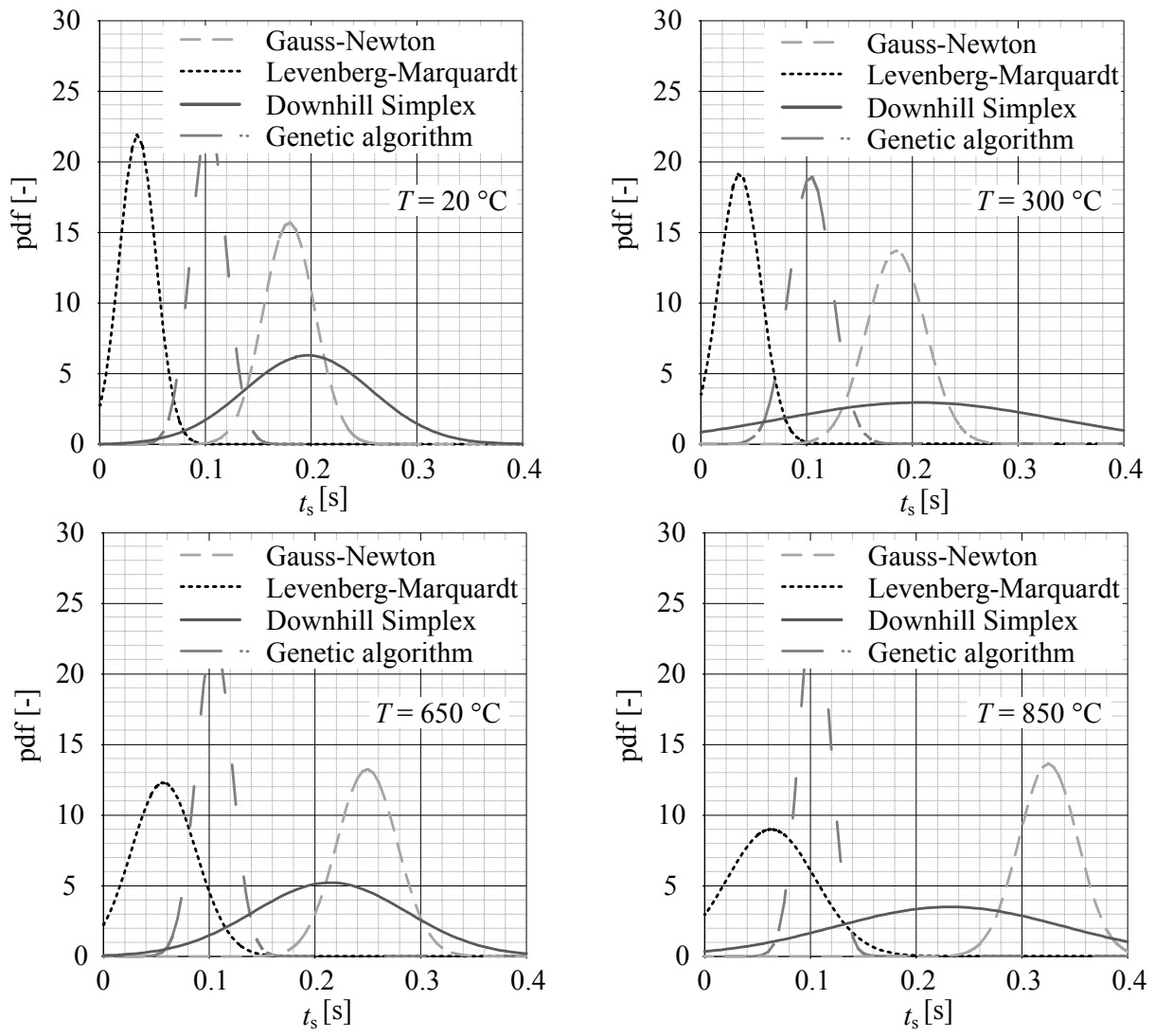

Figure 6. Processing time per search for the optimal set of material parameters $R_{0}, Q_{\infty}$ and $\gamma$. 


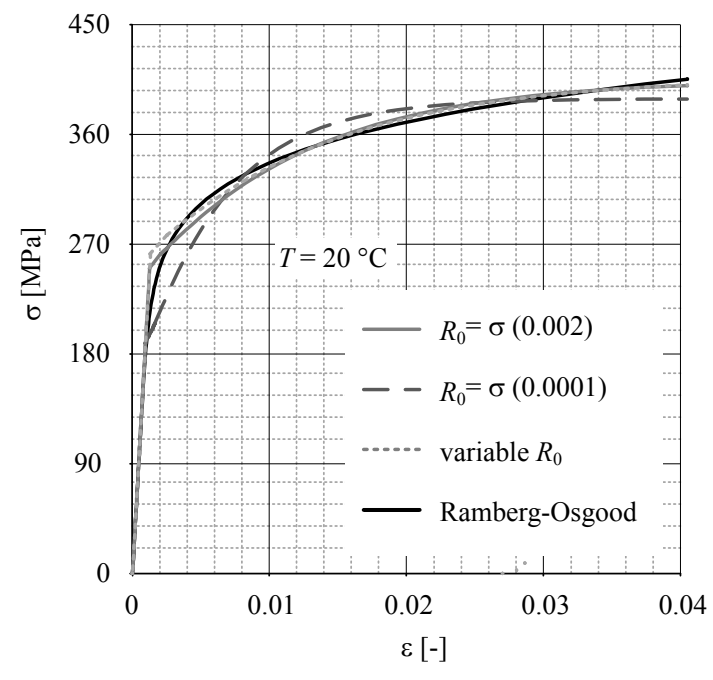

Figure 7. Influence of changing the parameter $R_{0}$.

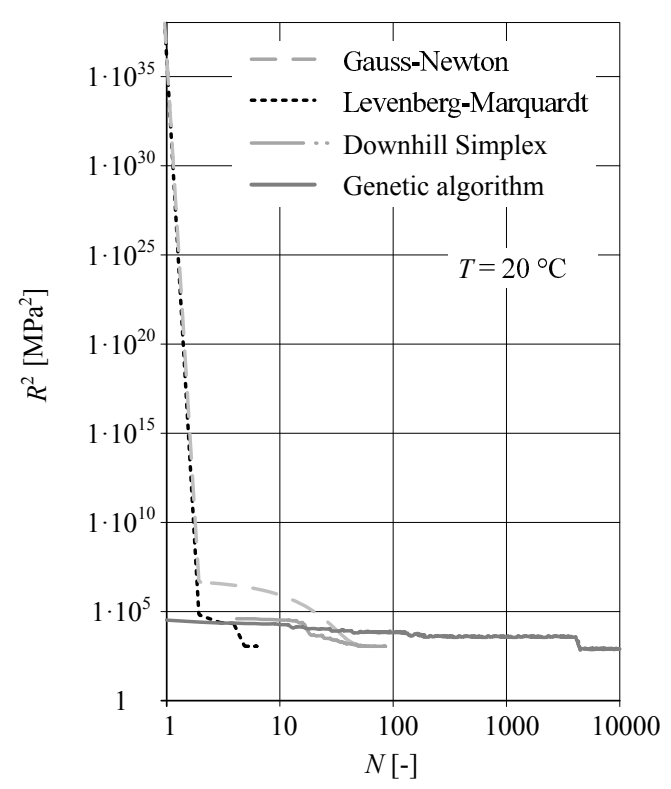

Figure 8. $R^{2}$ value dependence.

time of the LM method can become comparable to those of the GA method as is observed at $850{ }^{\circ} \mathrm{C}$ in Fig. 6 .

The GN method is computationally slowest and needs the largest number of steps to reach the optimum. As with the LM method the computational time to reach the optimal values of the material parameters depends on the shape of the curve as can be seen from Fig. 6. The number of steps and the computational time can be adjusted by changing the value of parameter $\beta$ in Eq. 9, but this way the GN method can start diverging and misses the optimal set of parameters. The GA method is always the most time-consuming overall, although the time needed to evaluate a generation of chromosomes is comparable to the fastest of the methods regardless of the temperature, i.e. the shape of the cyclic curve (Fig. 6). Overall slowness of the GA method thus comes from the fact that all the pre-set steps have to be performed (10000 steps in the example) whereas the other methods can stop once the convergence criterion has been met (Figs. 5 and 8). Although there exist other versions of the GA method, this does not influence the general properties of the method which have been compared here. The DS method shows the biggest scatter in both quantities, the number of steps to reach the convergence and the time to perform a search. This is influenced by the initial position and the shape of the simplex. Larger initial simplexes in general require more steps to reach the optimum than smaller simplexes closer to the optimal solution. A visual comparison of the search paths in Fig. 4 also shows the "character" of the methods. The GN method smoothly shifts from the initial to the final state. On the contrary, the LM method descends towards the optimum with rough movements. The DS method quickly drops into the valley and then manoeuvres towards the goal whereas the GA method usually approaches the valley during the first generation of chromosomes and then oscillates towards and around the optimum with the amplitude proportional to the mutation rate.

A possibility for a global optimum after knowing the method-dependent final sets of optimal values of material parameter vectors $\mathbf{x}_{\mathrm{gn}}^{\mathrm{opt}}=\left\{\begin{array}{c}R_{0} \\ Q_{\infty} \\ \gamma\end{array}\right\}_{\mathrm{gn}}^{\mathrm{opt}}$, $\mathbf{x}_{\operatorname{lm}}^{\mathrm{opt}}=\left\{\begin{array}{c}R_{0} \\ Q_{\infty} \\ \gamma\end{array}\right\}_{\mathrm{lm}}^{\mathrm{opt}}, \mathbf{x}_{\mathrm{ds}}^{\mathrm{opt}}=\left\{\begin{array}{c}R_{0} \\ Q_{\infty} \\ \gamma\end{array}\right\}_{\mathrm{ds}}^{\mathrm{opt}}$ and $\mathbf{x}_{\mathrm{ga}}^{\mathrm{opt}}=$ $\left\{\begin{array}{c}R_{0} \\ Q_{\infty} \\ \gamma\end{array}\right\}_{\mathrm{ga}}^{\mathrm{opt}}$ can be a choice of that particular set which will give the minimal values of the objective function among all methods.

\section{Conclusion}

Cyclic curves are comparable for all the tested temperatures of ferritic stainless steel EN 1.4512 regardless of the optimisation method utilised. The LM and the GN methods always find the optimal set of values of the material parameters in the smallest and the highest number of steps, respectively. The DS method shows the largest scatter of both the number of steps and the computational time. The GA method is the most time-consuming of the methods tested here.

\section{Acknowledgements}

This paper is a part of research work within program $\mathrm{Nr}$. P2-0182 entitled Development evaluation financed by the Slovenian Ministry of Education, Science and Sport. The authors are very grateful for the financial support.

\section{References}

1. Nayebi A and Hamidpour M. Thermo-mechanical cyclic loading analysis of pipes with different type of defects: Temperature dependent properties. Proceedings of the Institution of Mechanical Engineers, Part L: Journal of Materials: Design and Applications 2016; 230(1): 303-310.

2. Nazir MH, Khan ZA, Saeed A et al. A predictive model for life assessment of automotive exhaust mufflers subject to internal 
corrosion failure due to exhaust gas condensation. Engineering Failure Analysis 2016; 63: 43-60.

3. Šeruga D, Hansenne E, Haesen V et al. Durability prediction of EN 1.4512 exhaust mufflers under thermomechanical loading. International Journal of Mechanical Sciences 2014; 84: 199207.

4. Dondapati S, Trivedi M, Dondapati RS et al. Investigation on the mechanical stresses in a muffler mounting bracket using root cause failure analysis (rcfa), finite element analysis and experimental validation. Engineering Failure Analysis 2017; 81: $145-154$.

5. Ghosh N, Pal PK and Nandi G. Gmaw dissimilar welding of aisi 409 ferritic stainless steel to aisi 3161 austenitic stainless steel by using aisi 308 filler wire. Engineering Science and Technology, an International Journal 2017; 20: 1334-1341.

6. Lakshminarayanan AK and Balasubramanian B. Understanding the parameters controlling friction stir welding of AISI 409M ferritic stainless steel. Metals and Materials International 2011; 17(6): 969-981.

7. Li MC, Zhang H, Huang RF et al. Effect of so 2 on oxidation of type 409 stainless steel and its implication on condensate corrosion in automotive mufflers. Corrosion Science 2014; 80: 96-103.

8. Andrade-Campos A, Teixeira-Dias F, Krupp U et al. Effect of strain rate, adiabatic heating and phase transformation phenomena on the mechanical behaviour of stainless steel. Strain 2010; 46: 283-297.

9. Nagode M, Šeruga D, Hack M et al. Damage operator-based lifetime calculation under thermomechanical fatigue and creep for application on uginox f12t en 1.4512 exhaust downpipes. Strain 2012; 48: 198-207.

10. Nagode M, Hack M and Fajdiga M. Low cycle thermomechanical fatigue: Damage operator approach. Fatigue Fract Engng Mater Struct 2010; 33: 149-160.

11. Dahlberg M and Segle P. Evaluation of models for cyclic plastic deformation a literature study. Technical report, Report number: 2010:45 - www.stralsakerhetsmyndigheten.se, 2010.

12. Dettmer $\mathrm{W}$ and Reese S. On the theoretical and numerical modelling of Armstrong-Frederick kinematic hardening in the finite strain regime. Computer Methods in Applied Mechanics and Engineering 2004; 193(1): 87-116.

13. Hales R, Holdsworth SR, ODonnell MP et al. A Code of Practice for the determination of cyclic stress-strain data. Materials at high temperatures 2002; 19(4): 165-185.

14. Lee MG and Kim SJ. Elastic-plastic constitutive model for accurate springback prediction in hot press sheet forming. Metals and Materials International 2012; 18(3): 425-432.

15. Ramberg W and Osgood WR. Description of stress-strain curves by three parameters. Technical report, NACA-TN-902, 1943.

16. Behroozinia P, Mirzaeifar R and Taheri S. A review of fatigue and fracture mechanics with a focus on rubber-based materials. Proceedings of the Institution of Mechanical Engineers, Part L: Journal of Materials: Design and Applications 2017; NA: NA.

17. Lopes JP and Malcher L. Implicit implementation of chaboche cyclic plasticity model. In CILAMCE 2015 Proceedings of the XXXVI Iberian Latin-American Congress on Computational Methods in Engineering.

18. O'Nora N. Compendium of thermoviscoplasticity modeling parameters for materials under nonisothermal fatigue. HIM 1990-2015. 1732 http://stars.library.ucf.edu/honorstheses1990-2015/1732, 2015.

19. Zaletelj H, Fajdiga G and Nagode M. Numerical methods for TMF cycle modeling. Stroj vestn-J Mech E 2011; 57: 485-494.

20. Bromesson R and Ekh M. Experiments and Modelling of the Cyclic Behaviour of Haynes 282. Technische Mechanik 2012; 32(2-5): 130-145.

21. Chaparro BM, Thuillier S, Menezes LF et al. Material parameters identification: Gradient-based, genetic and hybrid optimization algorithms. Computational Materials Science 2008; 44(2): 339-346.

22. Gong YP, Hyde CJ, Sun W et al. Determination of material properties in the Chaboche unified viscoplasticity model. Proceedings of the Institution of Mechanical Engineers, Part L: Journal of Materials: Design and Applications 2010; 224(1): 19-29.

23. Gorash Y and Mackenzie D. Safe structural design for fatigue and creep using cyclic yield strength. In ECCC - Creep and Fracture Conference 2014.

24. Kabir SMH and Yeo T. Material parameters of a structural steel with plastic strain memory. Journal of Mechanics Engineering and Automation 2011; 1: 10-17.

25. Maciejewski L, Myszka W and Ziętek G. Application of differential evolution algorithm for identification of experimental data. The archive of Mechanical Engineering 2007; 54(4): 328-337.

26. Rossi B and Jaspart JP. Mechanical behavior of a ferritic stainless steel under simple and complex loading paths. In International Conference on Structure and Architecture : ICSA2010. pp. 175-176.

27. Wagner $M$ and Decker M. Simulation of thermo-mechanical deformation behavior and lifetime of a nickel-base alloy. Procedia Engineering 2015; 133: 272-281.

28. Armstrong PJ and Frederick CO. A mathematical representation of the multiaxial bauschinger effect. Technical report, Report RD/B/N731, CEGB, Central Electricity Generating Board, Berkley, UK., 1966.

29. Jiang Y and Kurath P. Characteristics of the ArmstrongFrederick type plasticity models. International Journal of Plasticity 1996; 12(3): 387-415.

30. Jirasek M and Bazant ZP. Inelastic Analysis of Structures. John Wiley \& Sons, 2002.

31. Seddik R, Sghaier RB and Fathallah R. A numericalanalytical approach to predict the effects of shot peening on the fatigue performance of the nickel-based super alloy waspaloy. Proceedings of the Institution of Mechanical Engineers, Part L: Journal of Materials: Design and Applications 2016; DOI: https://doi.org/10.1177/1464420716663030.

32. Li L, Zhang G and Khandelwal K. Design of energy dissipating elastoplastic structures under cyclic loads using topology optimization. Structural and Multidisciplinary Optimization 2017; 56: 391-412.

33. Nagode $M$ and Zingsheim F. An online algorithm for temperature influenced fatigue-life estimation: strain-life approach. Int J Fatigue 2004; 26: 151-161.

34. Nagode M, Längler F and Hack M. A time-dependent damage operator approach to thermo-mechanical fatigue of Ni-resist D5S. International Journal of Fatigue 2011; 33: 692 - 699.

35. Šeruga D and Nagode M. Unification of the most commonly used time-temperature creep parameters. Materials Science \& Engineering A 2011; 528: 2804 - 2811. 
36. Valente RAF, Andrade-Campos A, Carvalho JF et al. Parameter identification and shape optimization. Optimization and Engineering 2011; 12(1-2): 129-152.

37. Radim Halama R, Markopoulos A and Sedlák J. Calibration of Chaboche model with a memory surface. Transactions of the VŠB Technical University of Ostrava, Mechanical Series 2013; 59(1): 1929.

38. Kłosowski $\mathrm{P}$ and Mleczek A. Parameters identification of Perzyna and Chaboche viscoplastic models for aluminum alloy at temperature of 120 C. Engineering Transactions 2014; 62(3): 291-305.

39. Franulovic M, Basan R and Krizan B. Kinematic hardening parameters identification with respect to objective function. International Journal of Mechanical, Aerospace, Industrial, Mechatronic and Manufacturing Engineering 2014; 8(4): 686690.

40. Armstrong JC and Favorite JA. Using a derivative-free optimization method for multiple solutions of inverse transport problems. Optim Eng 2016; 17: 105-125.

41. García-Palacios J, Castro C and Samartín A. Optimal design in elasticity: a systematic adjoint approach to boundary cost functionals. Optim Eng 2015; 16: 811-829.

42. Akkaram S, Beeson $\mathrm{D}$, Agarwal $\mathrm{H}$ et al. Inverse modeling technology for parameter estimation. Structural and Multidisciplinary Optimization 2007; 34: 151-164.

43. Booth RF, Bormotov DY and Borovik AV. Genetic algorithms and equations in free groups and semigroups. Contemporary Mathematics 2004; 349: 63-80.

44. Garbowski T, Maier G and Novati G. On calibration of orthotropic elastic-plastic constitutive models for paper foils by biaxial tests and inverse analyses. Structural and Multidisciplinary Optimization 2012; 46: 111-128.

45. Gavin HP. The levenberg-marquardt method for nonlinear least squares curve-fitting problems. Department of Civil and Environmental Engineering, Duke University, 2017.

46. Haupt RL and Haupt SE. Practical Genetic Algorithms. John Wiley \& Sons, 2004.

47. Hermawanto D. Genetic algorithm for solving simple mathematical equality problem. Indonesian Institute of Sciences (LIPI), 2013.

48. Levenberg K. A method for the solution of certain non-linear problems in least squares. Quarterly of Applied Mathematics 1944; 2(2): 164-168.

49. Madsen K, Nielsen HB and Tingleff O. Methods for non-linear least squares problems. Technical University of Denmark, 2004.

50. Marquardt DW. An algorithm for least-squares estimation of nonlinear parameters. Journal of the Society for Industrial and Applied Mathematics 1963; 11(2): 431-441.

51. Nelder JA and Mead R. A simplex method for function minimization. Comput J 1965; 7(4): 308-313.

52. Škrlec A and Klemenc J. Parameter identification for a cowpersymonds material model using a genetic algorithm combined with a response surface. Engineering Computations 2017; 34(3): 921-940.

53. Press WH, Teukolsky SA, Vetterling WT et al. Numerical recipes: The art of scientific computing. Cambridge University Press, 2002. 